\title{
Role of Matriptase-2 (TMPRSS6) in Iron Metabolism
}

\author{
Pauline Lee \\ Scripps Research Institute, La Jolla, Calif., USA
}

\section{Key Words}

CUB · Hemojuvelin · Hepcidin • Iron · LDLa $\cdot$ Matriptase TMPRSS $\cdot$ Type II serine protease

\begin{abstract}
Iron, an essential element for life, is regulated primarily at the level of uptake, storage, and transport in order to maintain sufficient availability for normal physiology. The key protein in iron homeostasis is a 25 -amino-acid peptide, hepcidin, which modulates the amount of iron in the circulation by binding and promoting the degradation of the iron exporter ferroportin. Given the central importance of hepcidin, recent studies have focused on how iron is sensed and how the iron signal is transmitted to hepcidin. Mutations in a type II serine protease, matriptase-2/TMPRSS6, were recently identified to be associated with severe iron deficiency caused by inappropriately high levels of hepcidin expression. A key biologically relevant substrate for the proteolytic activity of matriptase-2/TMPRSS6 was found to be hemojuvelin, a cell surface protein that regulates hepcidin expression through a BMP/SMAD pathway. In this review, we discuss the putative role of matriptase-2/TMPRSS6 in iron homeostasis.
\end{abstract}

Copyright $\odot 2009$ S. Karger AG, Basel

\section{Introduction}

Recent identification of the importance of TMPRSS6/ matriptase-2 in iron homeostasis has resulted in the publication of several excellent review articles [1-3]. This review is written as a special tribute to Dr. Ernest Beutler, for whom the understanding of iron homeostasis was one of his many research passions. It will include work done in his laboratory the final year of his life and shortly afterwards, and will discuss some aspects of the field not covered by previous review articles.

Matriptase-2, also called TMPRSS6, is a type II plasma membrane serine protease (TTSP). The 811-amino-acid (aa) human protein is synthesized as an inactive zymogen and autoactivated by proteolytic cleavage. Structurally, matriptase- 2 contains a short 54 -aa N-terminal cytoplasmic domain, a membrane-spanning region, an SEA (sea urchin sperm protein, enteropeptidase, and agrin) domain, 2 CUB [Cls/Clr, urchin embryonic growth factor, and bone morphogeneic protein (BMP)-1] domains, three LDLa (low-density-lipoprotein receptor, class A) domains, and a trypsin-like serine protease domain containing the catalytic triad of histidine, aspartate, and serine residues (fig. 1). Matriptase-2 contains 38 conserved cysteine residues predicted to form 18 extracellular intramolecular disulfide bonds, three of which are within the catalytic protease domain (C593-C609, C724-C738, and C749-C778). One of the disulfide bonds (C559-C679)

\section{KARGER}

(ㄷ) 2009 S. Karger AG, Basel

Fax +41613061234 E-Mail karger@karger.ch www.karger.com
Pauline Lee, $\mathrm{PhD}$

Department of Molecular and Experimental Medicine, Scripps Research Institute 10550 North Torrey Pines Road, MEM 215

La Jolla, CA 92037 (USA)

Tel. +1 858784 2217, Fax +1 858784 2083, E-Mail plee@ scripps.edu 


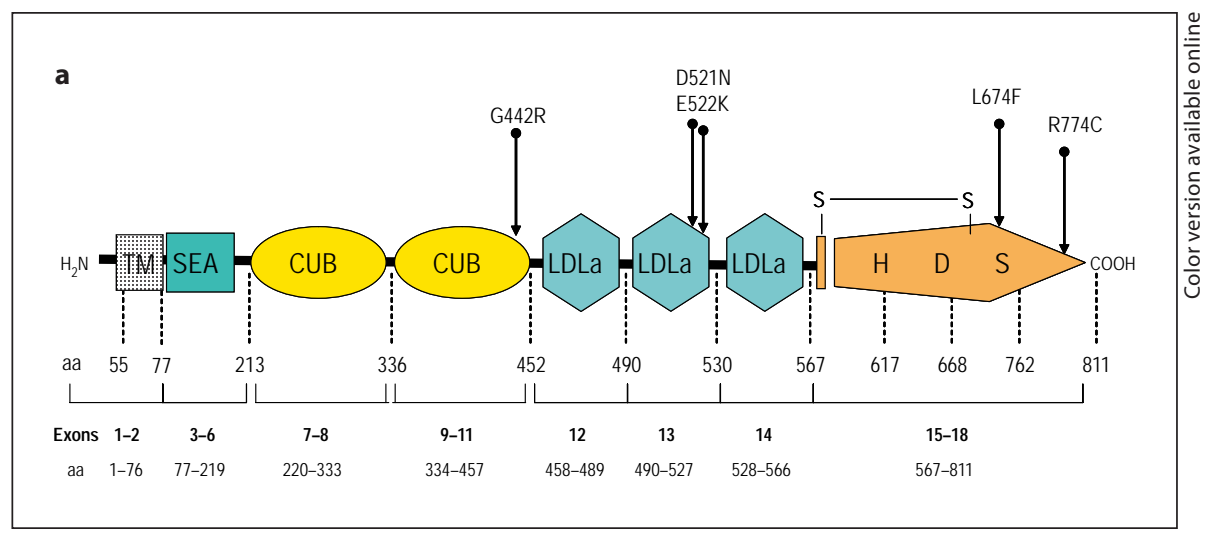

Fig. 1. Schematic model of matriptase-2. a Structurally, matriptase- 2 contains a short N-terminal cytoplasmic domain, a membrane-spanning region (TM), an SEA domain, 2 CUB domains, three LDLa domains, and a trypsin-like serine protease domain with the highly conserved catalytic triad of histidine (H617), aspartic acid (D668), and serine (S762) residues. Coding region mutations resulting in an aa change that have been identified in IRIDA patients are indicated by arrows [17, 20, 30]. b Schematic model of matriptase-2 truncation mutants identified in IRIDA patients [17-20, 30].

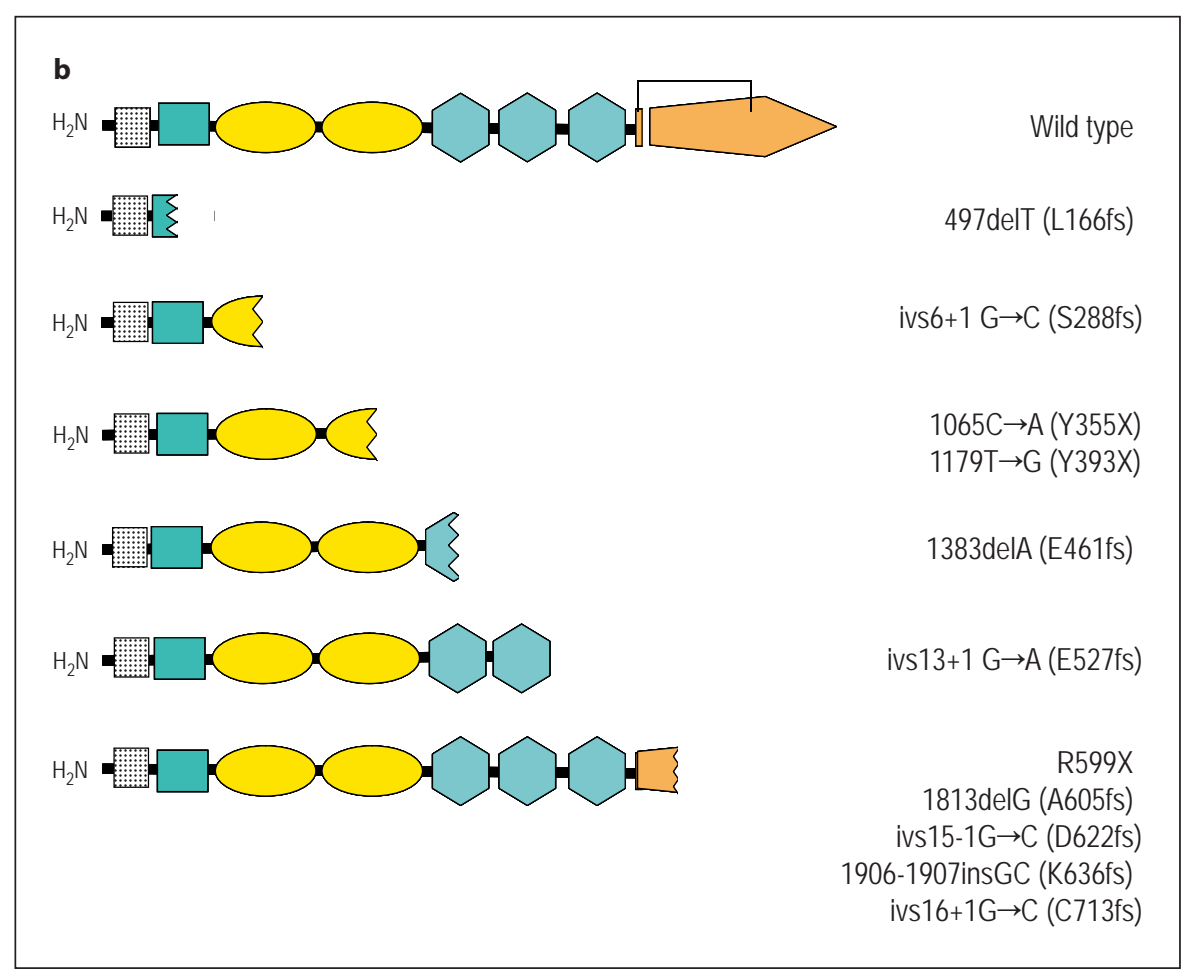

serves to hold the protease domain to the stem region after proteolytic autoactivation. The unpaired cysteine residue in the SEA domain and the unpaired intracellular cysteine residue have the potential to participate in intermolecular bonding. Matriptase-2 lacks group A scavenger receptor domains representative of other members of the hepsin/TMPRSS subfamily and lacks frizzled domains present in the corin subfamily of TTSPs [1]. The differences in protein structure and differences in aa sequence homology between the TMPRSS and matriptase subfamilies suggest that TMPRSS6 is a misnomer and its designation as matriptase- 2 is more accurate [1]. For this reason, for the remainder of this review, the protein will be referred to as matriptase-2, but the gene will be referred to as TMPRSS6.

SEA domains are $O$-glycosylated and may play a role in binding to other cell surface glycoproteins or substrate proteins. Within the SEA domains of several membrane proteins is a conserved cleavage site, GSVIV (enteropeptidase), GSVIA (matriptase), and GSVVV (mucin) by which proteolytic processing after the glycine residue sheds the protein from the cell surface. Matriptase-2, which has been reported to be shed from the cell surface [4], contains the motif GSLRV in the homologous region of the SEA domain that might be the putative cleavage site. 
Table 1. Matriptase-2 protein and synthetic peptide substrates

\begin{tabular}{|c|c|c|}
\hline \multicolumn{3}{|c|}{ Matriptase-2 cleavage of synthetic peptides } \\
\hline Artificial & Boc QAR $\downarrow-A m c$ & {$[8]$} \\
\hline Artificial & Boc QNR $\downarrow-A m c$ & {$[8]$} \\
\hline Filaggrin & $\mathrm{RKR} \downarrow \mathbf{R} \downarrow G S R G$ & {$[12]$} \\
\hline Trask (CDCP1) & KQR\SKFVP & {$[12]$} \\
\hline$\alpha_{\mathrm{E}} \beta_{7}$ Integrin & RQRR $\downarrow A L E K$ & {$[12]$} \\
\hline \multicolumn{3}{|c|}{ Matriptase-2 cleavage of proteins } \\
\hline TMPRSS6 autocleavage & PSSR $\downarrow I V G G$ & {$[8]$} \\
\hline Hemojuvelin (furin site) & RNRRGAIT & {$[4 ; 13]$} \\
\hline Fibronectin & single site & {$[8]$} \\
\hline Type I collagen & single site & {$[8]$} \\
\hline Fibrinogen & single site & {$[8]$} \\
\hline
\end{tabular}

Protein substrates and synthetic peptide substrates are listed. The cleavage sites are indicated with arrows $(\downarrow)$. The aa sequences of fibronectin, collagen, and fibrinogen do not reveal an obvious site that would be a target for matriptase- 2 .

CUB domains are found in mostly developmentally regulated proteins. Cubulin, which contains 27 CUB domains, has been shown to bind transferrin, hemoglobin, albumin, intrinsic factor-vitamin $\mathrm{B}_{12}$, vitamin-D-binding protein, apolipoprotein $\mathrm{A}_{1}$, and high-density lipoprotein, and participates in the processing of many of these proteins [5]. Binding of transferrin and hemoglobin to cubulin has been associated with transport into endosomes and lysosomes [5]. The CUB domain of platelet and endothelial cell surface protein SCUBE1 has been shown to bind BMP2 and plays a role in the secretion of mature BMP2 into the media [6]. The metalloproteinase ADAMTS13 contains two CUB domains, of which naturally occurring disease-associated mutations in the CUB1 domain have been identified. The mutations in the CUB1 domain of ADAMTS13 impaired secretion and stability of the secreted protease [7]. Of the two CUB domains in matriptase-2, the first one is degenerate relative to consensus CUB domains [8]. Nevertheless, it is likely that the CUB domains in matriptase- 2 play a role in its own cell surface localization, and in substrate binding and processing. It has not been elucidated whether the CUB domains in matriptase- 2 are able to bind transferrin or BMP2, two relevant proteins in iron homeostasis.

LDLa domains are cysteine-rich domains of approximately 40 aa with a conserved D/NXSDE motif of which the aspartic acid and glutamic acid residues are involved in calcium binding. LDLa domains have been found in a variety of functionally unrelated proteins and apparently play a role in the binding, internalization, and processing of proteins [9-11]. To date, matriptase-2 has not been shown to participate in the internalization of macromolecules, including substrates or substrate cleavage products, or mediate the processing of substrates within endocytic compartments, observed with some LDL receptor family members. Furthermore, it has not been determined if internalization or binding to matriptase- 2 induces a signaling event.

\section{Matriptase-2 Substrates}

Autoactivation of matriptase- 2 is predicted to occur by cleaving a conserved RIVGG motif located in the activation domain [8]. Matriptase-2 has been shown to cleave fibronectin, type 1 collagen, fibrinogen, pro-urokinase plasminogen activator, and artificial substrates with the peptide recognition sequence QAR and QGR [8] (table 1). Studies comparing substrate specificities of matriptase-2 to matriptase-1, hepsin and DESC1 demonstrated that matriptase- 2 was the most promiscuous of the four proteases [12]. Matriptase-2 mediated efficient cleavage of artificial peptides corresponding to cleavage sites located in the proteins filaggrin, CUB-domain-containing protein 1 (CDCP1; also called transmembrane and associated with src kinases, Trask), and $\alpha_{\mathrm{E}} \beta_{7}$ integrin [12] (table 1). Using internally quenched fluorescent peptides as substrates, matriptase- 2 demonstrated a preference for arginines in the $\mathrm{P} 1$ position and a basic aa in the $\mathrm{P} 4$ position. Matriptase-2 accommodates glycine, serine, alanine, isoleucine, or arginine in the $\mathrm{P}^{\prime}$ position (table 1).

Hemojuvelin was the first biologically relevant exogenous substrate for matriptase-2 to be identified [4] (table 1). Coexpression of matriptase- 2 with hemojuvelin resulted in the generation of several 25 - to $30-\mathrm{kDa}$ fragments and the reduction of all soluble hemojuvelin cleavage products at high concentrations of matriptase-2. Examination of the hemojuvelin sequence reveals the presence of a furin cleavage motif RNRR $\downarrow G$ that closely resembles the matriptase cleavage motif for filaggrin (RKR $\downarrow R \downarrow G S R G)$. Nevertheless, the hemojuvelin R335Q mutant that alters the furin cleavage motif of hemojuvelin to RNRQG is cleaved in the same manner as wild-type hemojuvelin [4]. Although it is conceivable that matriptase- 2 cleaves between arginine and glutamine of the R335Q mutant hemojuvelin (RNR $\downarrow$ Q) since the artificial peptide BocQNR-Amc is cleaved efficiently, the presence of a negatively charged aa (glutamic acid) at $\mathrm{P} 4$ and the glutamine at $\mathrm{P1}^{\prime}$ argue against this hypothesis [12]. Fur- 


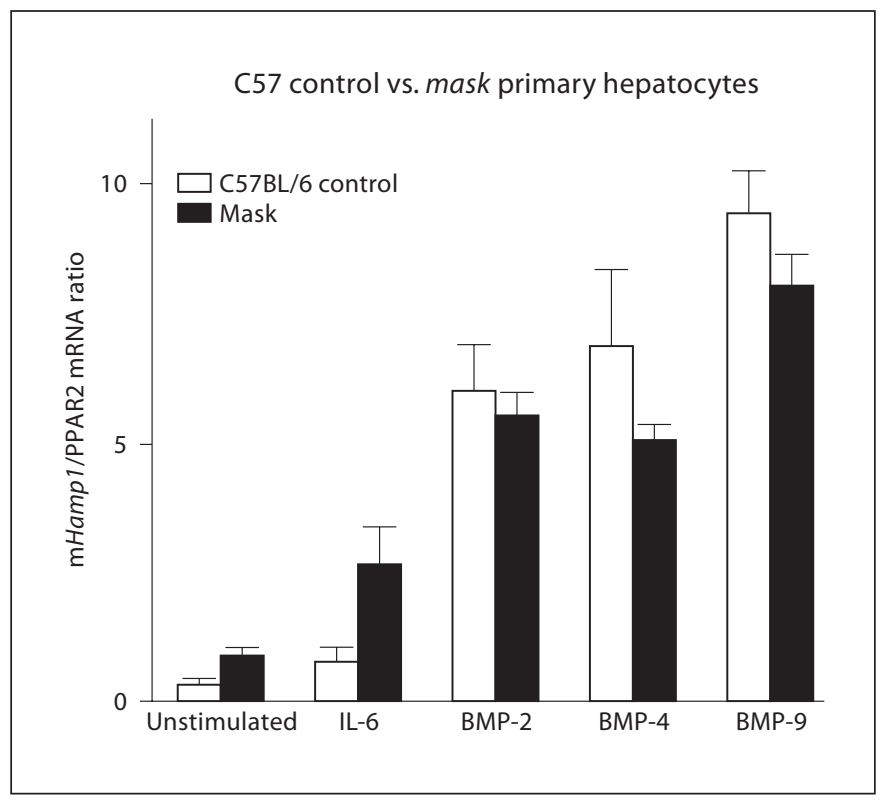

Fig. 2. Responsiveness of endogenous Hamp1 mRNA expression to IL- 6 and BMPs. Endogenous Hampl expression was measured in primary hepatocytes isolated from matriptase-2 mask mutant or C57BL/6 control mice and stimulated with or without $10 \mathrm{ng} / \mathrm{ml}$ of IL-6, BMP2, BMP4, or BMP9. The normalization gene used is $\mathrm{S} 18$ ribosomal protein (PPAR2, GenBank accession No. AK050626). Each bar represents the mean \pm SEM of two or three experiments each performed in triplicate.

thermore, the cleavage products of hemojuvelin mediated by matriptase- 2 appear to differ from cleavage products of hemojuvelin mediated by furin [13]. Proteomic studies need to be performed to identify the sites of cleavage of hemojuvelin to determine if matriptase-2-mediated cleavage products are identical to previously described 'shed' hemojuvelin [14].

\section{Matriptase-2 and Iron Homeostasis}

The importance of matriptase-2 in iron homeostasis was first demonstrated by Du et al. [15] in mutant mask mice (MGI:3776631), generated by N-ethyl-N-nitrosourea mutagenesis [16]. Mask mice named for their truncal alopecia exhibited microcytic anemia, low plasma iron levels, low spleen iron stores, and high levels of Hamp1 expression, inappropriate in the context of iron deficiency anemia [16]. The high expression of Hamp1 mRNA, encoding the 25-aa iron-regulatory peptide hepcidin, accounted for the reduced intestinal iron absorption ob- served in these mice [16]. Mask mice do not lower Hampl mRNA levels in response to an iron-deficient diet as do C57 control mice [16]. Surprisingly, mask mice also do not increase Hamp1 mRNA levels in response to a highiron diet presumably because the higher than normal Hamp1 mRNA levels reflect that mice are already 'seeing' an apparently high iron state [31]. Nevertheless, high dietary or parenteral iron levels are able to correct the low hemoglobin levels, alopecia, and female infertility in Tmprss6 mask mutant and null mice [16, 32]. Although Hamp1 mRNA expression is unresponsive to iron in mask mice, Hamp1 mRNA expression is responsive to stimulation by BMP2, BMP4, and BMP9, and to IL-6 (fig. 2).

Overexpression of wild-type or mutant (mask or the S762A) matriptase-2 in HepG2 cells demonstrated that wild-type but not mutant matriptase-2 repressed Hamp1 reporter expression induced by BMP2, BMP9, IL-6, and hemojuvelin [16]. Thus, the in vitro data and the mask mouse phenotype supported that matriptase- 2 was a potent inhibitor of Hampl mRNA expression.

Overexpression of mutant (mask or R774C) matriptase-2 in zebrafish resulted in defective hemoglobin production suggesting a dominant negative effect over endogenous wild-type matriptase-2 [4]. Overexpression of wild-type matriptase-2 in zebrafish did not affect hemoglobin production, but it is not known whether the fish exhibited decreased Hamp1 mRNA levels, increased ferroportin levels, and an iron overload phenotype compared to control fish [4].

The discovery of severe iron deficiency anemia in mask mice led to the identification of mutations in TMPRSS6 in human patients with iron-refractory iron deficiency anemia (IRIDA) [17-20, 30]. In both mice and humans, the iron deficiency associated with mutations in TMPRSS6 showed a recessive mode of transmission. Since the Tmprss6-/- mice (Tmprss6 ${ }^{\text {tmlOtin }}$, MGI:3809291) exhibited the identical iron deficiency phenotype, it is unlikely that mutations in TMPRSS6 are associated with a gain-in-function phenotype. Tmprss6-/- mice exhibited reduced ferroportin immunostaining and iron accumulation in intestinal enterocytes consistent with a phenotype expected by overexpression of hepcidin [32].

The identification of hemojuvelin as a key substrate for matriptase- 2 provided an explanation for the iron deficiency anemia associated with mutations in the gene for matriptase-2 (TMPRSS6) [4]. Cell surface hemojuvelin is correlated with hepcidin expression in hepatocytes [21]. Hemojuvelin is a coreceptor for some BMPs, in particular 
BMP6, and participates in the activation of the SMAD1/ SMAD4 pathway to upregulate transcription of the hepcidin gene (HAMP) $[22,23,33]$. Deficiency in hemojuvelin, associated with juvenile hemochromatosis in humans, results in abnormally low expression of hepcidin leading to severe iron overload [24]. In matriptase-2 deficiency, hemojuvelin expression is presumably elevated leading to increased hepcidin expression, yet this remains to be demonstrated in vivo. Nevertheless, if this is the case, it is important to note that matriptase-2-deficient mice are not hyperresponsive to stimulation by BMP2, BMP4, or BMP9 (fig. 2).

Immunoprecipitation studies demonstrated physical association of matriptase-2 with hemojuvelin [4]. Binding to hemojuvelin occurred independently of the protease domain since the mask mutant matriptase-2 was also able to bind hemojuvelin. Coexpression of wild-type matriptase-2 and hemojuvelin in Hep3B or HeLa cells demonstrated cleavage of hemojuvelin. Matriptase-2 lacking or defective in the protease domain was unable to cleave hemojuvelin. Membrane-associated matriptase- 2 does not cleave soluble hemojuvelin, and soluble matriptase does not cleave membrane-associated hemojuvelin. The data suggest that coexpression of matriptase-2 and membrane-associated hemojuvelin is required for cleavage [4].

In an effort to determine the phenotype of mice lacking both functional matriptase- 2 and hemojuvelin, a double-mutant mouse strain $\mathrm{Hfe}^{\text {tmINca/tmlNca}} ; \mathrm{Tmprss}^{\text {Msk/Msk }}$ was generated [31]. Mice lacking both matriptase-2 and hemojuvelin exhibited high plasma iron, high transferrin saturation, and normal hemoglobin and mean corpuscular volume, high liver iron content, and low Hampl mRNA levels, similar to hemojuvelin null mice. These data demonstrated that in the absence of both the positive and negative stimuli of hemojuvelin and matriptase-2, respectively, Hamp1 expression was constitutively off. Furthermore, Hamp1 mRNA expression in mice lacking both matriptase- 2 and hemojuvelin was responsive to BMP2, BMP4, and BMP9, and the inflammatory cytokine IL-6, but was not responsive to high dietary iron. Therefore, mice lacking both matriptase- 2 and hemojuvelin, being highly similar to hemojuvelin null mice, support the model that hemojuvelin is a substrate for matriptase-2. If matriptase- 2 were acting on a substrate downstream of hemojuvelin, an iron-deficient or normal phenotype rather than an iron overload phenotype would have resulted.

Matriptase-2 and Iron Metabolism

\section{Mutations and Polymorphisms in Matriptase-2}

Mutations in matriptase- 2 have provided insight into the mechanism of action of matriptase-2. Mutations associated with severe iron deficiency in mice or human patients predominately affect the proteolytic activity of matriptase-2 (fig. 1). Severe mutations in the protease domain of matriptase- 2 causing premature termination and leading to an iron deficiency phenotype include the splice site mutation in mask mice (ivs14-2 A $\rightarrow \mathrm{G}$; C566fs) which deletes the entire protease domain, an R599X point mutationidentified in zorro mice (Bruce Beutler, Tmprss6 ${ }^{\mathrm{m} 2 \mathrm{Btl}}$, MGI:3812005) [25] and in an IRIDA patient [18], the W783X point mutation in masquerade mice (Bruce Beutler, Tmprss6 ${ }^{\mathrm{m} 3 \mathrm{Btlr}}$, MGI:3829007) [26], two deletion/insertion mutations A605fs, K636fs, and a splice site mutation (ivs16+1 G $\rightarrow$ C; G713fs) identified in IRIDA patients [17]. In addition, the point mutation identified in an IRIDA patient R774C located in the protease domain likely disrupts accurate $\mathrm{C} 32 / \mathrm{C} 36$ or $\mathrm{C} 35 / \mathrm{C} 37$ disulfide bonding within the protease domain [17]. Furthermore, the protease dead mutation, S762A in matriptase-2, has been shown in vitro to be ineffective in repressing Hamp1 reporter expression [16].

Point mutations D521N and E522K located in the conserved D/NXSDE motif in the LDLa2 domain of matriptase-2 have been associated with IRIDA in patients and affect residues predicted to bind $\mathrm{Ca}^{2+}[17,20]$. In vitro, the $\mathrm{D} 521 \mathrm{~N}$ and $\mathrm{E} 522 \mathrm{~K}$ mutants show reduced cell surface localization, increased Golgi retention, impaired autoactivation of matriptase-2, impaired cleavage of hemojuvelin, and impaired ability to repress Hamp1 reporter expression but are able to bind hemojuvelin [20].

Matriptase-2 containing the point mutation G442R located in the second CUB domain demonstrated impaired autoactivation, was still able to bind but demonstrated reduced cleavage of coexpressed hemojuvelin, and exhibited reduced ability to repress HAMP reporter expression [20].

The mutants L166fs and S288fs result in severe truncation of the ectodomain and if they localize to the cell surface, they are likely to be severely impaired in their ability to bind hemojuvelin. These mutations identified in homozygous IRIDA patients suggest that loss of proteolytic cleavage of hemojuvelin rather than increased stabilization or retention of hemojuvelin on the cell surface by mutant matriptase- 2 is sufficient to cause the IRIDA phenotype. Furthermore, these data demonstrate that homozygous loss of the bulk of the ectodomain does not induce

Acta Haematol 2009;122:87-96 
Table 2. List of coding region polymorphisms in the human TMPRSS6 gene

\begin{tabular}{|c|c|c|c|c|c|c|}
\hline SNP ID & $\begin{array}{l}\text { Genomic } \\
\text { coordinates }\end{array}$ & $\begin{array}{l}\text { Contiguous } \\
\text { coordinates }\end{array}$ & Alleles & $\begin{array}{l}\text { Coding } \\
\text { coordinates }\end{array}$ & $\mathrm{AA}$ & $\begin{array}{l}\text { NCBI allele } \\
\text { frequency }\end{array}$ \\
\hline & 129 & 16890080 & $\mathrm{C} / \mathrm{T}$ & 15 & F5F & \\
\hline rs11704654 & 308 & 16889901 & $\mathrm{~A} / \mathrm{G}$ & 99 & P33P & 0.299 \\
\hline \multirow[t]{2}{*}{ rs5756514 } & 5198 & 16885011 & $\mathrm{C} / \mathrm{T}$ & 323 & S108F & ND \\
\hline & 13896 & 16876313 & $\mathrm{~A} / \mathrm{G}$ & 683 & G228D* & \\
\hline rs35961386 & 13903 & 16876306 & $\mathrm{C} / \mathrm{T}$ & 690 & G230G & ND \\
\hline rs2235324 & 13970 & 16876239 & $\mathrm{~A} / \mathrm{G}$ & 757 & K253E & 0.496 \\
\hline rs5995378 & 14076 & 16876133 & $\mathrm{C} / \mathrm{T}$ & 863 & S288L & 0.034 \\
\hline rs2543517 & 17358 & 16872851 & $\mathrm{G} / \mathrm{A}$ & 987 & P329P & ND \\
\hline rs2111833 & 18897 & 16871312 & $\mathrm{G} / \mathrm{A}$ & 1083 & S361S & 0.452 \\
\hline \multirow[t]{2}{*}{ rs881144 } & 28404 & 16861805 & $\mathrm{C} / \mathrm{T}$ & 1254 & Y418Y & ND \\
\hline & 28486 & 16861723 & $\mathrm{C} / \mathrm{T}$ & 1336 & R446W* & \\
\hline rs4820268 & 30103 & 16860106 & $\mathrm{C} / \mathrm{T}$ & 1563 & D521D & 0.488 \\
\hline rs855791 & 36758 & 16853451 & $\mathrm{~T} / \mathrm{C}$ & 2207 & V736A & 0.416 \\
\hline rs2235321 & 36768 & 16853441 & $\mathrm{C} / \mathrm{T}$ & 2217 & Y739Y & 0.471 \\
\hline rs11703011 & 36826 & 16852783 & $\mathrm{G} / \mathrm{A}$ & 2288 & G763D & ND \\
\hline \multirow[t]{2}{*}{ rs73886915 } & 36884 & 16852725 & $\mathrm{C} / \mathrm{T}$ & 2346 & S782S & ND \\
\hline & 37521 & 16852688 & $\mathrm{~A} / \mathrm{G}$ & 2383 & V795I* & ND \\
\hline
\end{tabular}

Coding region polymorphisms identified in the NCBI SNP database, or by screening $\left(^{*}\right)$ of subjects with or without iron deficiency anemia of unknown etiology [30]. ND = Not determined.

an intracellular signal leading to repression of hepcidin expression, as previously proposed [16].

Thus most, if not all of the disease-associated mutations in TMPRSS6, result in the loss of proteolytic activity by loss of the protease domain or reduction in autoactivation, or in a reduction in cell surface localization.

The identification of severe mutations in matriptase-2 associated with IRIDA raised the question as to whether mild mutations or polymorphisms might contribute to iron deficiency anemia in patients lacking predisposing factors. Examination of the TMPRSS6 gene revealed that the gene was highly polymorphic, particularly in noncoding regions. Coding region polymorphisms occurring in the general population (table 2) did not appear to correlate with iron deficiency anemia of no known etiology, although the allele frequencies of rare polymorphisms located in highly conserved regions of the protein were too low to establish statistical significance [30]. Nevertheless, in one family study, the presence of an uncommon R446W polymorphism in trans with a severe TMPRSS6 mutation appeared to be associated with ironresponsive iron deficiency anemia. Functional studies need to be performed to determine if these rare polymor- phisms in matriptase-2 exhibit impaired hemojuvelin degradation and impaired suppression of Hampl expression.

\section{Hemojuvelin Shedding}

How does liver-specific matriptase-2 fit into the earlier reports of soluble hemojuvelin in cell culture supernatants, serum, and plasma [21]? Previously, the release of hemojuvelin into cell culture media was shown to be suppressed by transferrin-bound iron or ferric ammonium citrate [21]. Soluble hemojuvelin is biologically active as a competitive inhibitor that can antagonize the binding of BMPs to the BMP receptor [21]. Is the cleavage of hemojuvelin by matriptase- 2 sensitive to iron? Are the products of hemojuvelin by matriptase- 2 cleavage able to antagonize BMP binding? When HeLa cells are cotransfected with matriptase- 2 and hemojuvelin, multiple cleavage products of hemojuvelin and loss of soluble hemojuvelin are observed [4], suggesting that the function of matriptase-2 is to degrade hemojuvelin rather than to produce an antagonist, but this remains to be examined in greater experimental detail. 
Kuninger et al. [27], Zhang et al. [28], and Lin et al. [21] demonstrated independently that hemojuvelin is shed from muscle cells (C2C12), transfected liver (HepG2 and Hep3B), and kidney cells (HEK293), strongly suggesting that there are matriptase-2-dependent and -independent pathways for shedding hemojuvelin since matriptase- 2 is almost exclusively expressed in the liver. Kuninger et al. [27] demonstrated that cell surface biotin-labeled hemojuvelin could be shed from the cell surface. The shed hemojuvelin was primarily of the form uncleaved at the 172Asp-173Pro bond. Lin et al. [14] demonstrated that hemojuvelin released from transfected HEK293 cells was cleaved by a furin-like proprotein convertase at the furin site and included both 172Asp-173Pro-cleaved and uncleaved forms of hemojuvelin. In the presence of inhibitors of furin-like proteases, hemojuvelin was shed from the cell surface by an alternative pathway, possibly by the action of phopholipase A, cleaving the glycosylphosphatidylinositol anchor and releasing the entire hemojuvelin ectodomain [14].

Silvestri et al. [13] demonstrated that mutants of hemojuvelin that were retained in the endoplasmic reticulum still released soluble hemojuvelin despite reduced cell surface localization, suggesting that soluble hemojuvelin was secreted from the endoplasmic reticulum rather than shed from the cell surface. Furthermore, they also provided evidence that cleavage of hemojuvelin was mediated by the protease furin which was transcriptionally regulated by iron and hypoxia. Cotransfection of HeLa and HepG2 cells with furin and hemojuvelin did not appear to show the same cleavage products as cotransfection with matriptase-2 and hemojuvelin [13].

Zhang et al. [28] indicated that hemojuvelin shedding is regulated by neogenin. Coexpression of hemojuvelin with neogenin in HepG2 cells increased hemojuvelin shedding. Likewise, knockdown of endogenous neogenin in $\mathrm{C} 2 \mathrm{C} 12$ cells transfected with hemojuvelin suppressed hemojuvelin shedding. Coexpression of hemojuvelin with neogenin in HEK293 cells was associated with increased iron loading [29].

The data seem to indicate that there are several pathways by which hemojuvelin can be released from cells, more than one of which may be sensitive to iron regulation. If this is the case, they clearly are not redundant since they do not compensate for the loss of matriptase-2 in humans and mice. Matriptase-2-independent shedding of hemojuvelin does not result in a major change in cell surface levels of hemojuvelin $[13,28]$ and appears to function to generate a soluble antagonist that can act in an autocrine or paracrine fashion depending on the tis- sue source, be it hepatocytes or muscles. In contrast, matriptase-2-dependent cleavage results in a major loss of hemojuvelin from the hepatocyte cell surface [4], and its function appears to be removal of the positive effect cellassociated hemojuvelin has on hepcidin expression. Removal of cell-associated hemojuvelin has a dominant phenotype over the antagonistic function of soluble hemojuvelin.

\section{Transcriptional Regulation of Matriptase-2}

The transcriptional regulation of matriptase- 2 might provide insight into mediators of matriptase-2 expression that indirectly effect iron homeostasis. Comparison of the genomic organization and nucleotide sequences of exons 1, 5'-untranslated regions and proximal promoter regions of the human and murine TMPRSS6 genes demonstrated that these regions are not conserved between species (fig. 3). Examination of the predicted transcription factor binding sites by Genomatix MatInspector (http://www.genomatix.com) identified a krueppel-like $\mathrm{C} 2 \mathrm{H} 2$ zinc finger factor hypermethylated in cancer (HIC1) motif, a peroxisome proliferator-activated receptor (PPAR_RXR) motif, a nuclear receptor subfamily 2 factor (COUP) motif, a pancreas transcription factor 1 , a heterotrimeric transcription factor (PTFI) motif, and an NKX homeodomain factor (NKXH) motif in common between human and murine proximal promoters, but currently there is no known biological relevance for any of these transcription factors in the regulation of matriptase-2 expression (fig. 3).

Since hepcidin expression is repressed by hypoxia and anemia, it raises the question whether matriptase- 2 plays a role in this repression. Studies need to be performed to examine if transcriptional and posttranscriptional regulation of matriptase- 2 by hypoxia or iron deficiency might contribute indirectly to the repression of hepcidin.

\section{Summary and Future Perspectives}

The current working model for the role of matriptase2 in iron homeostasis has membrane-anchored matriptase- 2 as an active protease acting as the central modulator of hemojuvelin cell surface expression (fig. 4a). Cleavage of hemojuvelin results in loss from the hepatocyte cell surface and the loss of hemojuvelin to act as a coreceptor for BMPs. Nevertheless, BMPs can still upregulate hepci- 


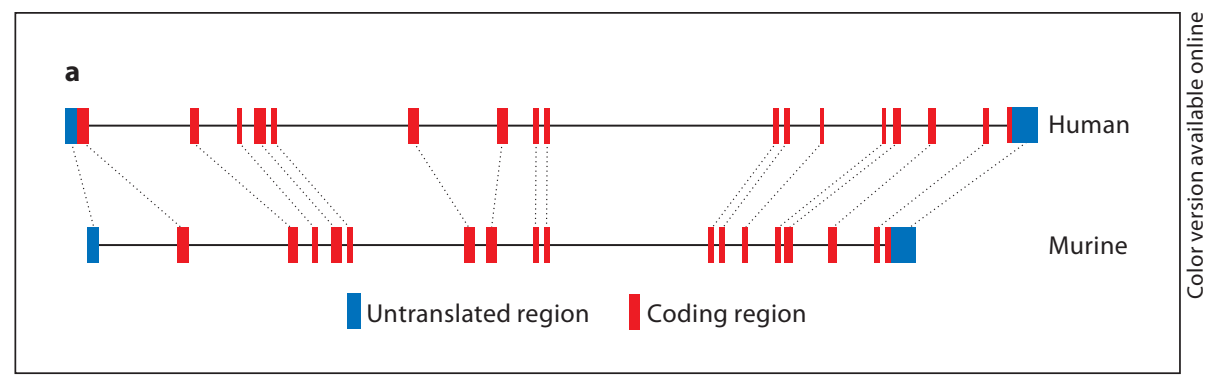

Fig. 3. Analysis of the human and murine TMPRSS6 genes. a Alignment of the genomic organization of the human and murine TMPRSS6 genes. b Genomatix MatInspector predicted transcription factor motifs common in the proximal promoter regions of the human and murine TMPRSS6 genes. The genomic organization at the $5^{\prime}$ ends of the human and murine genes is not conserved. There is little to no sequence homology between the human and murine promoter and $5^{\prime}$-untranslated regions. Non-coding genomic coordinates are used (numbering from the ATG). b

Human promoter

-494 cgaaatgaat attagttttt gttccettcc tctgccacaa gactttgaga gcagaaaggt -434 gagagagacg gtactctgtg aaggaaggca ggtccceggc ccagcgcagt gccagctcag NKXH

-374 gggattctgg ggcggggget aagtgcatgg actgtgtggg cgtggtggga agctccgtga

PPAR RxR/COUP/PTF1

-314 accagaacca ggagcaagaa acagcattcc ttgcgtggac gggaāatgag ggcaagaggt

HIC1

-254 cagatgtcta cagaagtctg caccccatgt acttcagttc tgtctgtggg tgcagcctct

-194 agggaggtgg gtgtttaggt actgagacct ccgtctgtcc tctgaccata gggaagccag

-134 tgggaagcaa aggtggggtt CTTGAGCCAG ACCCAGTCCA GCTCTGGTGC CTGCCCTCTG

-74 GTGCGAGCTG ACCTGAGATG CACTTCCCTC CTCTGTGAGC TGTCTCGGCA CCCACTTGCA

-14 GTCACTGCCG CCTG

Mouse promoter

NKXH

-529 aagagagtgt caagtgcacc tctcccceg cccacttctc tcctatcccc agaagtcacg

-469 ggcccaggac tttctggcca gtgtcagctg tgtgagcccc tgttctcacc tgaatctgcc

-409 tctgcagttt cccattcacc ctgttagggg accagggget gtaaccaggc aggaatatca

PPAR RXR/COUP/PTF1

-349 ccaagcaaca ggcccogggg gagagctcag gtctcetgct cetgcaggtc cagcccctg

HIC1

-289 ggcacccatg gggcagggeg gggttagaga ggcgggeact aactacttgc ctcctgcctc -229 ctgcctgttt acctgtgttc agtttcattg tcgccetgga cctGACAGGA GAGGCCCATG

-169 GAACTTGGGG CCACAGGCCA CAAGGGACAA GGGCCAGACA CCCCAGCCAT GGCTCCAGGC

-109 CATTGATCCA ACCTAAGCTG GCCAGTTGGg GGTGGAAAGA CCTTGGCCTG GATAAACAGA

- 49 GGCCTCCAGG CCTGTGTGCA GGCCCGGCAC CTACCTTCCA CTCTTGAAG din expression directly through the BMP receptors in the absence of hemojuvelin. Likewise, inflammatory cytokines such as IL- 6 are able to upregulate hepcidin expression in the absence of hemojuvelin. It remains to be determined if matriptase-2-mediated cleavage products of hemojuvelin are able to act as a soluble antagonist for the binding of BMPs to the cognate receptor in the same manner as soluble hemojuvelin [21]. The matriptase-2 mask mutant lacking the protease domain is able to localize to the cell surface and can bind hemojuvelin. Uncleaved hemojuvelin is stable and retained on the cell sur- face where it is perpetually available to promote BMPmediated activation of hepcidin expression. The presence of the matriptase-2 mask mutant does not interfere with IL-6-mediated upregulation of hepcidin expression (fig. 4b).

There are so many questions left to answer, many of which were raised throughout the manuscript. Is matriptase-2 activity regulated by iron? What roles do TfR2, HFE, and neogenin play, if any, to relay the iron status to matriptase-2? If matriptase- 2 is not regulated by iron then what is it regulated by - hypoxia? Are there multiple 
Fig. 4. Working model of matriptase-2 regulation of iron homeostasis. a Membraneanchored matriptase-2 (MTP2) is an active protease cleaving hemojuvelin $(\mathrm{HJV})$ resulting in loss of hemojuvelin, a coreceptor for BMPs, from the hepatocyte cell surface. Nevertheless, BMPs can still upregulate hepcidin expression directly through binding to the BMP receptors in the absence of hemojuvelin. Likewise, inflammatory cytokines such as IL- 6 are able to upregulate hepcidin expression in the absence of hemojuvelin. It remains to be determined if matriptase-2-mediated cleavage products of hemojuvelin are able to act as a soluble antagonist for the binding of BMPs to the cognate receptor in the same manner as soluble hemojuvelin (sHJV). b The matriptase-2 mask (MTP2 $2^{\text {mask }}$ ) mutant lacking the protease domain is able to localize to the cell surface and can bind hemojuvelin. Uncleaved hemojuvelin is stable and retained on the cell surface where it is perpetually available to promote BMPmediated activation of hepcidin expression. The presence of the matriptase-2 mask mutant does not interfere with IL-6mediated upregulation of hepcidin expression.
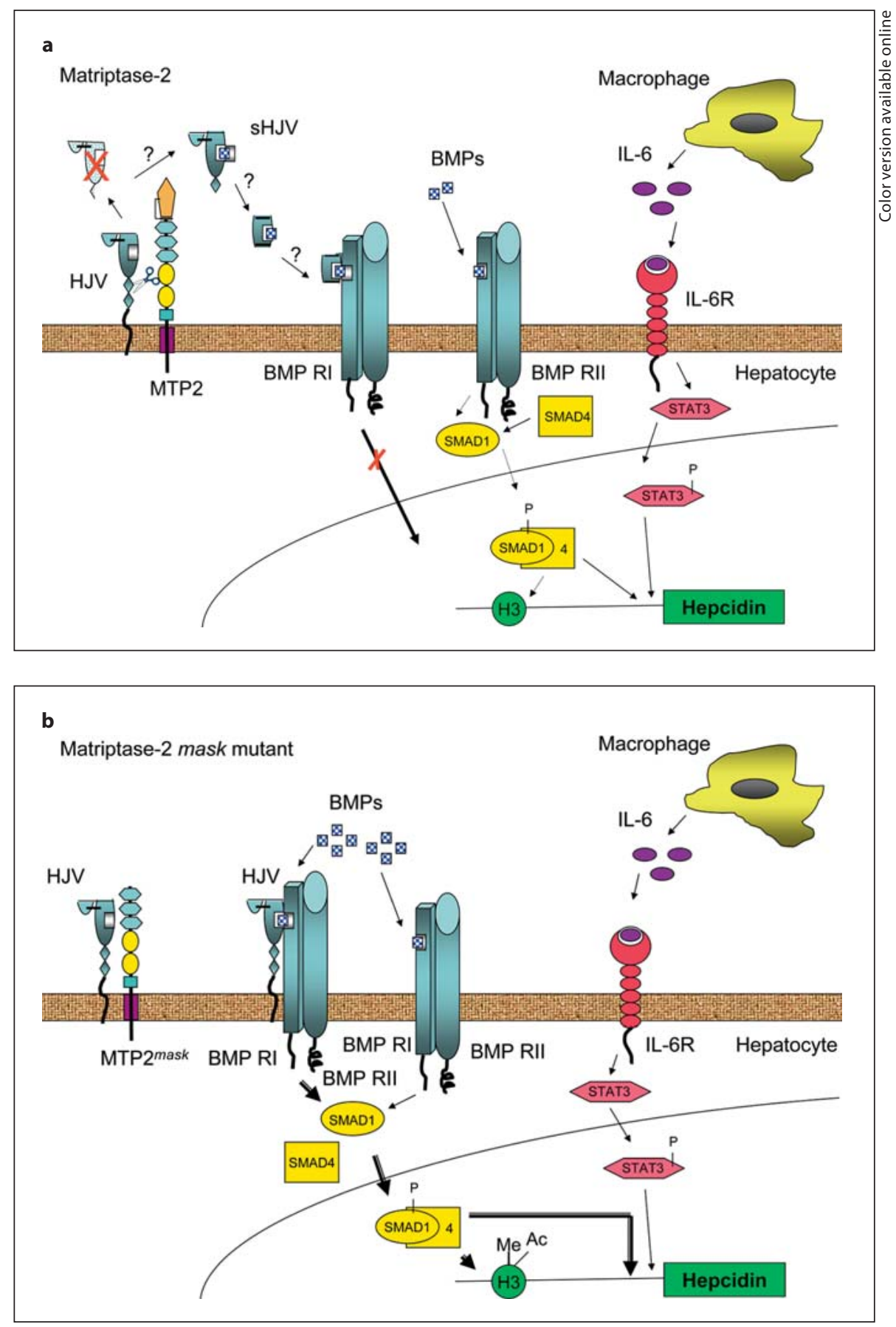

pathways for cleaving hemojuvelin - a shedding pathway to produce a soluble antagonist and another to degrade cell surface hemojuvelin?

Are there other biologically important substrates for matriptase-2? Does soluble matriptase-2 (shed from the cell surface) have physiological importance?

\section{Acknowledgments}

This work (manuscript 20141-MEM from the Scripps Research Institute) was supported by grants from the National Institutes of Health (grant DK53505-10), the Skaggs Scholars in Clinical Science Program from the Scripps Research Institute and the Stein Endowment Fund. 


\section{References}

-1 Ramsay AJ, Hooper JD, Folgueras AR, Velasco G, Lopez-Otin C: Matriptase-2 (TMPRSS6): a proteolytic regulator of iron homeostasis. Haematologica 2009;94:840849.

2 Knutson MD: Into the matrix: regulation of the iron regulatory hormone hepcidin by matriptase-2. Nutr Rev 2009;67:284-288.

-3 Muckenthaler MU: Fine tuning of hepcidin expression by positive and negative regulators. Cell Metab 2008;8:1-3.

-4 Silvestri L, Pagani A, Nai A, De Domenico I, Kaplan J, Camaschella C: The serine protease matriptase-2 (TMPRSS6) inhibits hepcidin activation by cleaving membrane hemojuvelin. Cell Metab 2008;8:502-511.

5 Christensen EI, Birn H: Megalin and cubilin: multifunctional endocytic receptors. Nat Rev Mol Cell Biol 2002;3:256-266.

- 6 Tu CF, Yan YT, Wu SY, Djoko B, Tsai MT, Cheng CJ, Yang RB: Domain and functional analysis of a novel platelet-endothelial cell surface protein, SCUBE1. J Biol Chem 2008; 283:12478-12488.

7 Zhou Z, Jing H, Tao Z, Choi H, Aboulfatova K, Moake J, Li R, Dong JF: Effects of naturally occurring mutations in CUB-1 domain on synthesis, stability, and activity of ADAMTS-13. Thromb Res 2009;124:323-327.

-8 Velasco G, Cal S, Quesada V, Sanchez LM, Lopez-Otin C: Matriptase-2, a membranebound mosaic serine proteinase predominantly expressed in human liver and showing degrading activity against extracellular matrix proteins. J Biol Chem 2002;277: 37637-37646.

9 Horn IR, van den Berg BM, Moestrup SK, Pannekoek H, van Zonneveld AJ: Plasminogen activator inhibitor 1 contains a cryptic high affinity receptor binding site that is exposed upon complex formation with tissuetype plasminogen activator. Thromb Haemost 1998;80:822-828.

10 Cam JA, Bu G: Modulation of beta-amyloid precursor protein trafficking and processing by the low density lipoprotein receptor family. Mol Neurodegener 2006;1:8.

- 11 Parkyn CJ, Vermeulen EG, Mootoosamy RC, Sunyach C, Jacobsen C, Oxvig C, Moestrup S, Liu Q, Bu G, Jen A, Morris RJ: LRP1 controls biosynthetic and endocytic trafficking of neuronal prion protein. J Cell Sci 2008; 121:773-783.

12 Beliveau F, Desilets A, Leduc R: Probing the substrate specificities of matriptase, matriptase-2, hepsin and DESC1 with internally quenched fluorescent peptides. FEBS J 2009; 276:2213-2226.
3 Silvestri L, Pagani A, Camaschella C: Furinmediated release of soluble hemojuvelin: a new link between hypoxia and iron homeostasis. Blood 2008;111:924-931.

$>14$ Lin L, Nemeth E, Goodnough JB, Thapa DR, Gabayan V, Ganz T: Soluble hemojuvelin is released by proprotein convertase-mediated cleavage at a conserved polybasic RNRR site. Blood Cells Mol Dis 2008;40:122-131.

$15 \mathrm{Du}$ X, Moresco EMY, Beutler B: Tmprss6 mask (updated 9-28-2008). La Jolla, Mutagenetix, accessed 5-10-09. http://mutagenetix.scripps.edu.

16 Du X, She E, Gelbart T, Truksa J, Lee P, Xia Y, Khovananth K, Mudd S, Mann N, Moresco EM, Beutler E, Beutler B: The serine protease TMPRSS6 is required to sense iron deficiency. Science 2008;320:1088-1092.

17 Finberg KE, Heeney MM, Campagna DR, Aydinok Y, Pearson HA, Hartman KR, Mayo MM, Samuel SM, Strouse JJ, Markianos K, Andrews NC, Fleming MD: Mutations in TMPRSS6 cause iron-refractory iron deficiency anemia (IRIDA). Nat Genet 2008;40: 569-571.

18 Guillem F, Lawson S, Kannengiesser C, Westerman M, Beaumont C, Grandchamp B: Two nonsense mutations in the TMPRSS6 gene in a patient with microcytic anemia and iron deficiency. Blood 2008;112:2089-2091.

19 Melis MA, Cau M, Congiu R, Sole G, Barella S, Cao A, Westerman M, Cazzola M, Galanello R: A mutation in the TMPRSS6 gene, encoding a transmembrane serine protease that suppresses hepcidin production, in familial iron deficiency anemia refractory to oral iron. Haematologica 2008;93:14731479.

20 Silvestri L, Guillem F, Pagani A, Nai A, Oudin C, Silva M, Toutain F, Kannengiesser C, Beaumont C, Camaschella C, Grandchamp B: Molecular mechanisms of the defective hepcidin inhibition in TMPRSS6 mutations associated with iron-refractory iron deficiency anemia. Blood 2009;113:56055608.

21 Lin L, Goldberg YP, Ganz T: Competitive regulation of hepcidin mRNA by soluble and cell-associated hemojuvelin. Blood 2005; 106:2884-2889.

22 Huang FW, Babitt JL, Wrighting DM, Samad TA, Xia Y, Sidis Y, Campagna JA, Chung RT, Schneyer AL, Woolf CJ, Andrews NC, Lin HY: Hemojuvelin acts as a bone morphogenetic protein co-receptor to regulate hepcidin. Blood 2005;106:153a.

23 Meynard D, Kautz L, Darnaud V, CanonneHergaux F, Coppin H, Roth MP: Lack of the bone morphogenetic protein BMP6 induces massive iron overload. Nat Genet 2009;41: 478-481.
24 Papanikolaou G, Samuels ME, Ludwig EH, MacDonald ML, Franchini PL, Dube MP, Andres L, MacFarlane J, Sakellaropoulos N, Politou M, Nemeth E, Thompson J, Risler JK, Zaborowska C, Babakaiff R, Radomski CC, Pape TD, Davidas O, Christakis J, Brissot P, Lockitch G, Ganz T, Hayden MR, Goldberg YP: Mutations in HFE2 cause iron overload in chromosome 1q-linked juvenile hemochromatosis. Nat Genet 2004;36:77-82.

$25 \mathrm{Du}$ X, Moresco EMY, Beutler B: Tmprss6 zorro (updated 9-28-2008). La Jolla, Mutagenetix, accessed 5-10-09. http://mutagenetix.scripps.edu.

26 Du X, Moresco EMY, Beutler B: Tmprss6 masquerade (9-28-2008). La Jolla, Mutagenix, accessed 5-10-09. http://mutagenetix. scripps.edu.

27 Kuninger D, Kuns-Hashimoto R, Kuzmickas R, Rotwein P: Complex biosynthesis of the muscle-enriched iron regulator RGMc. J Cell Sci 2006;119:3273-3283.

28 Zhang AS, Anderson SA, Meyers KR, Hernandez C, Eisenstein RS, Enns CA: Evidence that inhibition of hemojuvelin shedding in response to iron is mediated through neogenin. J Biol Chem 2007;282:12547-12556.

29 Zhang AS, West AP Jr, Wyman AE, Bjorkman PJ, Enns CA: Interaction of hemojuvelin with neogenin results in iron accumulation in human embryonic kidney 293 cells. J Biol Chem 2005;280:33885-33894.

30 Beutler E, Van Geet C, Te Loo DM, Gelbart T, Crain K, Truksa J, Lee PL: Polymorphisms and mutations of human TMPRSS6 in iron deficiency anemia. Blood Cells Mol Dis 2009; in press.

- 31 Truksa J, Gelbart T, Peng H, Beutler E, Beutler B, Lee P: Suppression of the hepcidinencoding gene Hamp permits iron overload in mice lacking both hemojuvelin and matriptase-2/TMPRSS6. Brit J Haematol DOI:10.1111/j.1365-2141.2009.07873x.

-32 Folgueras AR, de Lara FM, Pendas AM, Garabaya $\mathrm{C}$, Rodriguez F, Astudillo A, Bernal T, Cabanillas R, Lopez-Otin C, Velasco G: Membrane-bound serine protease matriptase-2 (Tmprss6) is an essential regulator of iron homeostasis. Blood 2008;112:25392545.

33 Andriopoulos B Jr, Corradini E, Xia Y, Faasse SA, Chen S, Grgurevic L, Knutson MD, Pietrangelo A, Vukicevic S, Lin HY, Babitt JL: BMP6 is a key endogenous regulator of hepcidin expression and iron metabolism. Nat Genet DOI:10.1038/ng.335. 